\title{
COMMON FIXED POINT THEOREMS IN 2 NON-ARCHIMEDEAN MENGER PM-SPACE
}

\author{
RENU CHUGH and SUMITRA
}

(Received 17 May 1999)

\begin{abstract}
We introduce the concept of a 2 non-Archimedean Menger PM-space and prove a common fixed point theorem for weak compatible mappings of type $(A)$.

2000 Mathematics Subject Classification. 47H10, 54H25.
\end{abstract}

1. Introduction. Cho et al. [1] proved a common fixed point theorem for compatible mappings of type $(A)$ in non-Archimedean (NA) Menger PM-space. The aim of this paper is to generalize the results of Cho et al. [1] for weak compatible mappings of type $(A)$ in a 2 NA Menger PM-space.

We first give some definitions and notations.

DEFINITION 1.1. Let $X$ be any nonempty set and $D$ the set of all left-continuous distribution functions. An ordered pair $(X, F)$ is said to be a 2 non-Archimedean probabilistic metric space (briefly 2 NA PM-space) if $F$ is a mapping from $X \times X \times X$ into $D$ satisfying the following conditions where the value of $F$ at $x, y, z \in X \times X \times X$ is represented by $F_{x, y, z}$ or $F(x, y, z)$ for all $x, y, z \in X$ such that

(i) $F_{x, y, z}(t)=1$ for all $t>0$ if and only if at least two of the three points are equal.

(ii) $F_{x, y, z}=F_{x, z, y}=F_{z, y, x}$.

(iii) $F_{x, y, z}(0)=0$.

(iv) If $F_{x, y, s}\left(t_{1}\right)=F_{x, s, z}\left(t_{2}\right)=F_{s, y, z}\left(t_{3}\right)=1$, then $F_{x, y, z}\left(\max \left\{t_{1}, t_{2}, t_{3}\right\}\right)=1$.

DEFINITION 1.2. A $t$-norm is a function $\Delta:[0,1] \times[0,1] \times[0,1] \rightarrow[0,1]$ which is associative, commutative, nondecreasing in each coordinate, and $\Delta(a, 1,1)=a$ for every $a \in[0,1]$.

DEFINITION 1.3. A 2 NA Menger PM-space is an ordered triplet $(X, F, \Delta)$, where $\Delta$ is a $t$-norm and $(X, F)$ is a 2 NA PM-space satisfying the following condition

$$
\begin{aligned}
& F_{x, y, z}\left(\max \left\{t_{1}, t_{2}, t_{3}\right\}\right) \\
& \quad \geq \Delta\left(F_{x, y, s}\left(t_{1}\right), F_{x, s, z}\left(t_{2}\right), F_{s, y, z}\left(t_{3}\right)\right) \quad \forall x, y, z \in X, t_{1}, t_{2}, t_{3} \geq 0 .
\end{aligned}
$$

DEFINITION 1.4. Let $(X, F, t)$ be a 2 NA Menger PM-space and $t$ a continuous $t$-norm, then $(X, F, t)$ is Hausdorff in the topology induced by the family of neighborhoods

$$
\left\{U_{x}\left(\epsilon, \lambda, a_{1}, a_{2}, \ldots, a_{n}\right) ; x, a_{i} \in X, \epsilon>0, i=1,2, \ldots, n, n \in \mathbb{Z}^{+}\right\},
$$

where $\mathbb{Z}^{+}$is the set of all positive integers and

$$
\begin{aligned}
U_{x}\left(\epsilon, \lambda, a_{1}, a_{2}, \ldots, a_{n}\right) & =\left\{y \in X ; F_{x, y, a_{i}}(\epsilon)>1-\lambda, 1 \leq i \leq n\right\} \\
& =\cap_{i=1}^{n}\left\{y \in X ; F_{x, y, a_{i}}(\epsilon)>1-\lambda, 1 \leq i \leq n\right\} .
\end{aligned}
$$


DeFinITION 1.5. A 2 NA Menger PM-space $(X, F)$ is said to be of type $(C)_{g}$ if there exists a $g \in \Omega$ such that

$$
g\left(F_{x, y, z}(t)\right) \leq \mathcal{g}\left(F_{x, y, a}(t)\right)+g\left(F_{x, a, z}(t)\right)+g\left(F_{a, y, z}(t)\right) \quad \forall x, y, z, a \in X, t \geq 0,
$$

where $\Omega=\{g \mid g:[0,1] \rightarrow[0, \infty)$ is continuous, strictly decreasing, $g(1)=0$, and $g(0)<\infty\}$.

DefinItion 1.6. A 2 NA Menger PM-space $(X, F, \Delta)$ is said to be of type $(D)_{g}$ if there exists a $g \in \Omega$ such that

$$
g\left(\Delta\left(t_{1}, t_{2}, t_{3}\right)\right) \leq g\left(t_{1}\right)+g\left(t_{2}\right)+g\left(t_{3}\right) \quad \forall t_{1}, t_{2}, t_{3} \in[0,1]
$$

REMARK 1.7. If 2 NA Menger PM-space $(X, F, \Delta)$ is of type $(D)_{g}$, then $(X, F, \Delta)$ is of type $(C) g$.

Throughout this paper, let $(X, F, \Delta)$ be a complete 2 NA Menger PM-space with a continuous strictly increasing $t$-norm $\Delta$. Let $\phi:[0, \infty) \rightarrow[0, \infty)$ be a function satisfying the condition $(\Phi) \phi$ is upper semi-continuous from right and $\phi(t)<t$ for all $t>0$.

LEMMA 1.8. If a function $\phi:[0, \infty) \rightarrow[0, \infty)$ satisfies the condition $(\Phi)$, then we get

(1) For all $t \geq 0, \lim _{n \rightarrow \infty} \phi^{n}(t)=0$, where $\phi^{n}(t)$ is the $n$th iteration of $\phi(t)$.

(2) If $\left\{t_{n}\right\}$ is a nondecreasing sequence of real numbers and $t_{n+1} \leq \phi\left(t_{n}\right), n=1,2, \ldots$. Then $\lim _{n \rightarrow \infty} t_{n}=0$. In particular, if $t \leq \phi(t)$ for all $t \geq 0$, then $t=0$.

LEMMA 1.9. Let $\left\{y_{n}\right\}$ be a sequence in $X$ such that $\lim _{n \rightarrow \infty} F_{y_{n}, y_{n+1}, a}(t)=1$ for all $t>0$. If the sequence $\left\{y_{n}\right\}$ is not a Cauchy sequence in $X$, then there exist $\epsilon_{0}>0, t_{0}>0$, and two sequences $\left\{m_{i}\right\}$ and $\left\{n_{i}\right\}$ of positive integers such that

(i) $m_{i}>n_{i}+1$ and $n_{i} \rightarrow \infty$ as $i \rightarrow \infty$.

(ii) $F_{y_{m_{i}}, y_{n_{i}}, a}\left(t_{0}\right)<1-\epsilon_{0}$ and $F_{y_{m_{i}-1}, y_{n_{i}}, a}\left(t_{0}\right) \geq 1-\epsilon_{0}, i=1,2, \ldots$

DeFinITION 1.10. Let $A, S: X \rightarrow X$ be mappings, $A$ and $S$ are said to be compatible if

$$
\lim _{n \rightarrow \infty} g\left(F_{A S x_{n}, S A x_{n}, a}(t)\right)=0 \quad \forall t>0, a \in X,
$$

when $\left\{x_{n}\right\}$ is a sequence in $X$ such that

$$
\lim _{n \rightarrow \infty} A x_{n}=z=\lim _{n \rightarrow \infty} S x_{n} \quad \text { for some } z \in X
$$

DeFinITION 1.11. Let $A, S: X \rightarrow X$ be mappings, $A$ and $S$ are said to be compatible of type $(A)$ if

$$
\lim _{n \rightarrow \infty} g\left(F_{A S x_{n}, S S x_{n}, a}(t)\right)=0=\lim _{n \rightarrow \infty} g\left(F_{S A x_{n}, A A x_{n}, a}(t)\right) \quad \forall t>0, a \in X,
$$

when $\left\{x_{n}\right\}$ is a sequence in $X$ such that

$$
\lim _{n \rightarrow \infty} A x_{n}=z=\lim _{n \rightarrow \infty} S x_{n} \quad \text { for some } z \in X
$$


DeFinItion 1.12. Let $A, S: X \rightarrow X$ be mappings, $A$ and $S$ are said to be weak compatible of type $(A)$ if

$$
\begin{aligned}
& \lim _{n \rightarrow \infty} g\left(F_{A S x_{n}, S S x_{n}, a}(t)\right) \geq \lim _{n \rightarrow \infty} g\left(F_{S A x_{n}, S S x_{n}, a}(t)\right), \\
& \lim _{n \rightarrow \infty} g\left(F_{S A x_{n}, A A x_{n}, a}(t)\right) \geq \lim _{n \rightarrow \infty} g\left(F_{A S x_{n}, A A x_{n}, a}(t)\right) \quad \forall t>0, a \in X,
\end{aligned}
$$

whenever $\left\{x_{n}\right\}$ is a sequence in $X$ such that

$$
\lim _{n \rightarrow \infty} A x_{n}=z=\lim _{n \rightarrow \infty} S x_{n} \quad \text { for some } z \in X .
$$

Proposition 1.13. Let $A, S: X \rightarrow X$ be continuous mappings. If $A$ and $S$ are compatible of type $(A)$, then they are weak compatible of type $(A)$.

Proof. Suppose that $A$ and $S$ are compatible of type $(A)$. Let $\left\{x_{n}\right\}$ be a sequence in $X$ such that

$$
\lim _{n \rightarrow \infty} A x_{n}=z=\lim _{n \rightarrow \infty} S x_{n} \quad \text { for some } z \in X
$$

then

$$
\begin{aligned}
\lim _{n \rightarrow \infty} g\left(F_{S A x_{n}, S S x_{n}, a}(t)\right) & =0 \\
& \leq \lim _{n \rightarrow \infty} g\left(F_{A S x_{n}, S S x_{n}, a}(t)\right) \\
& \Longrightarrow \lim _{n \rightarrow \infty} g\left(F_{A S x_{n}, S S x_{n}, a}(t)\right) \geq \lim _{n \rightarrow \infty} g\left(F_{S A x_{n}, S S x_{n}, a}(t)\right) .
\end{aligned}
$$

Similarly, we can show that

$$
\begin{aligned}
\lim _{n \rightarrow \infty} g\left(F_{S A x_{n}, A A x_{n}, a}(t)\right) & =0 \\
& \geq \lim _{n \rightarrow \infty} g\left(F_{A S x_{n}, A A x_{n}, a}(t)\right) .
\end{aligned}
$$

Therefore, $A$ and $S$ are weak compatible of type $(A)$.

Proposition 1.14. Let $A, S: X \rightarrow X$ be weak compatible mappings of type $(A)$. If one of $A$ and $S$ is continuous, then $A$ and $S$ are compatible of type $(A)$.

Proof. Let $\left\{x_{n}\right\}$ be a sequence in $X$ such that

$$
\lim _{n \rightarrow \infty} A x_{n}=z=\lim _{n \rightarrow \infty} S x_{n} \quad \text { for some } z \in X .
$$

Suppose $S$ is continuous so $S S x_{n}, S A x_{n} \rightarrow S z$ as $n \rightarrow \infty$. Since $A$ and $S$ are weak compatible of type $(A)$, so we have

$$
\begin{aligned}
\lim _{n \rightarrow \infty} g\left(F_{A S x_{n}, S S x_{n}, a}(t)\right) & \geq \lim _{n \rightarrow \infty} g\left(F_{S A x_{n}, S S x_{n}, a}(t)\right) \\
& =\lim _{n \rightarrow \infty} g\left(F_{S z, S z, a}(t)\right)=0 .
\end{aligned}
$$

Thus

$$
\lim _{n \rightarrow \infty} g\left(F_{A S x_{n}, S S x_{n}, a}(t)\right)=0 .
$$

Similarly,

$$
\lim _{n \rightarrow \infty} g\left(F_{S A x_{n}, A A x_{n}, a}(t)\right)=0 .
$$

Hence $A$ and $S$ are compatible of type $(A)$. 
Proposition 1.15. Let $A, S: X \rightarrow X$ be continuous mappings. Then $A$ and $S$ are compatible of type $(A)$ if and only if $A$ and $S$ are weak compatible of type $(A)$.

Note that Proposition 1.15 is a direct consequence of Propositions 1.13 and 1.14.

Proposition 1.16. Let $A, S: X \rightarrow X$ be mappings. If $A$ and $S$ are weak compatible of type $(A)$ and $A z=S z$ for some $z \in X$. Then $S A z=A A z=A S z=S S z$.

Proof. Suppose that $\left\{x_{n}\right\}$ is a sequence in $X$ defined by $x_{n}=z, n=1,2, \ldots$, and $A z=S z$ for some $z \in X$. Then we have $A x_{n}, S x_{n} \rightarrow S z$ as $n \rightarrow \infty$. Since $A$ and $S$ are weak compatible of type $(A)$ so

$$
\begin{aligned}
& \lim _{n \rightarrow \infty} g\left(F_{A S x_{n}, S S x_{n}, a}(t)\right) \geq \lim _{n \rightarrow \infty} g\left(F_{S A x_{n}, S S x_{n}, a}(t)\right), \\
& \lim _{n \rightarrow \infty} g\left(F_{S A x_{n}, A A x_{n}, a}(t)\right) \geq \lim _{n \rightarrow \infty} g\left(F_{A S x_{n}, A A x_{n}, a}(t)\right) .
\end{aligned}
$$

Now

$$
\begin{aligned}
\lim _{n \rightarrow \infty} \mathcal{g}\left(F_{S A z, A A z, a}(t)\right) & =\lim _{n \rightarrow \infty} \mathcal{g}\left(F_{S A x_{n}, A A x_{n}, a}(t)\right) \geq \lim _{n \rightarrow \infty} g\left(F_{A S x_{n}, A A x_{n}, a}(t)\right) \\
& =g\left(F_{A S z, S S z, a}(t)\right) .
\end{aligned}
$$

Since $S z=A z$, then $S A z=A A z$. Similarly, we have $A S z=S S z$. But $A z=S z$ for $z \in X$ implies that $A A z=A S z=S A z=S S z$.

Proposition 1.17. Let $A, S: X \rightarrow X$ be weak compatible mappings of type $(A)$ and let $\left\{x_{n}\right\}$ be a sequence in $X$ such that $\lim _{n \rightarrow \infty} A x_{n}=z=\lim _{n \rightarrow \infty} S x_{n}$ for some $z \in X$, then

(1) $\lim _{n \rightarrow \infty} A S x_{n}=S z$ if $S$ is continuous at $z$.

(2) $S A z=A S z$ and $A z=S z$ if $A$ and $S$ are continuous at $z$.

Proof. Suppose that $S$ is continuous and $\left\{x_{n}\right\}$ is a sequence in $X$ such that

$$
\lim _{n \rightarrow \infty} A x_{n}=z=\lim _{n \rightarrow \infty} S x_{n} \quad \text { for some } z \in X,
$$

so

$$
S S x_{n} \longrightarrow S z \quad \text { as } n \rightarrow \infty .
$$

Since $A$ and $S$ are weak compatible of type $(A)$, we have

$$
\begin{aligned}
g\left(F_{A S x_{n}, S z, a}(t)\right) & =\lim _{n \rightarrow \infty} g\left(F_{A S x_{n}, S S x_{n}, a}(t)\right) \\
& \geq \lim _{n \rightarrow \infty} g\left(F_{S A x_{n}, S S x_{n}, a}(t)\right) \longrightarrow 0 \quad \text { as } n \longrightarrow \infty
\end{aligned}
$$

for all $t>0$ which implies that $A S x_{n} \rightarrow S z$ as $n \rightarrow \infty$.

(2) Suppose that $A$ and $S$ are continuous at $z$. Since $A x_{n} \rightarrow z$ as $n \rightarrow \infty$ and $S$ is continuous at $z$, by Proposition 1.17(1) $A S x_{n} \rightarrow S z$ as $n \rightarrow \infty$. On the other hand, since $S x_{n} \rightarrow z$ as $n \rightarrow \infty$ and $A$ is also continuous at $z, A S x_{n} \rightarrow A z$ as $n \rightarrow \infty$. Thus $A z=S z$ by the uniqueness of the limit and so by Proposition 1.16, $S A z=A A z=A S z=S S z$. Therefore, we have $A S z=S A z$.

THEOREM 1.18. Let $A, B, S, T: X \rightarrow X$ be mappings satisfying

(i) $A(X) \subset T(X), B(X) \subset S(X)$, 
(ii) the pairs $A, S$ and $B, T$ are weak compatible of type $(A)$,

(iii) $S$ and $T$ is continuous,

(iv) $g\left(F_{A x, B y, a}(t)\right) \leq \phi\left(\max \left\{g\left(F_{S x, T y, a}(t)\right), g\left(F_{S x, A x, a}(t)\right), g\left(F_{T y, B y, a}(t)\right)\right.\right.$, $\left.\left.(1 / 2)\left(g\left(F_{S x, B y, a}(t)\right)+g\left(F_{T y, A x, a}(t)\right)\right)\right\}\right)$,

for all $t>0, a \in X$ where a function $\phi:[0, \infty) \rightarrow[0, \infty)$ satisfies the condition $(\Phi)$. Then by (i) since $A(X) \subset T(X)$, for any $x_{0} \in X$, there exists a point $x_{1} \in X$ such that $A x_{0}=T x_{1}$. Since $B(X) \subset S(X)$, for this $x_{1}$, we can choose a point $x_{2} \in X$ such that $B x_{1}=S x_{2}$ and so on, inductively, we can define a sequence $\left\{y_{n}\right\}$ in $X$ such that

$$
y_{2 n}=A x_{2 n}=T x_{2 n+1}, \quad y_{2 n+1}=B x_{2 n+1}=S x_{2 n+2}, \quad \text { for } n=0,1,2, \ldots
$$

First we prove the following lemma.

LEMMA 1.19. Let $A, S: X \rightarrow X$ be mappings satisfying conditions (i) and (iv), then the sequence $\left\{y_{n}\right\}$ defined by (1.24), such that

$$
\lim _{n \rightarrow \infty} g\left(F_{y_{n}, y_{n+1}}(t)\right)=0 \quad \forall t>0, a \in X,
$$

is a Cauchy sequence in $X$.

Proof. Since $g \in \Omega$, it follows that $\lim _{n \rightarrow \infty}\left(F_{y_{n}, y_{n+1}, a}(t)\right)=0$ for all $a>0, a \in X$ if and only if $\lim _{n \rightarrow \infty} g\left(F_{y_{n}, y_{n+1}, a}(t)\right)=0$ for all $t>0$. By Lemma 1.9, if $\left\{y_{n}\right\}$ is not a Cauchy sequence in $X$, there exist $\epsilon_{0}>0, t_{0}>0$, and two sequences $\left\{m_{i}\right\},\left\{n_{i}\right\}$ of positive integers such that

(A) $m_{i}>n_{i}+1$ and $n_{i} \rightarrow \infty$ as $i \rightarrow \infty$,

(B) $g\left(F_{y_{m_{i}}, y_{n_{i}}, a}\left(t_{0}\right)\right)>g\left(1-\epsilon_{0}\right)$ and $g\left(F_{y_{m_{i}}-1, y_{n_{i}}, a}\left(t_{0}\right)\right) \leq g\left(1-\epsilon_{0}\right), i=1,2, \ldots$, since $g(t)=1-t$. Thus we have

$$
\begin{aligned}
g\left(1-\epsilon_{0}\right) & <g\left(F_{y_{m_{i}}, y_{n_{i}}, a}\left(t_{0}\right)\right) \\
& \leq \mathcal{g}\left(F_{y_{m_{i}}, y_{n_{i}}, y_{m_{i}-1}}\left(t_{0}\right)\right)+g\left(F_{y_{m_{i}}, y_{m_{i}-1}}, a\left(t_{0}\right)\right)+g\left(F_{y_{m_{i}-1}, y_{n_{i}}, a}\left(t_{0}\right)\right) \\
& \leq \mathcal{g}\left(F_{y_{m_{i}}, y_{n_{i}}, y_{m_{i}-1}}\left(t_{0}\right)\right)+g\left(F_{y_{m_{i}}, y_{m_{i}-1}, a}\left(t_{0}\right)\right)+g\left(1-\epsilon_{0}\right) .
\end{aligned}
$$

As $i \rightarrow \infty$ in (1.26), we have

$$
\lim _{n \rightarrow \infty} g\left(F_{y_{m_{i}}, y_{n_{i}}, a}\left(t_{0}\right)\right)=g\left(1-\epsilon_{0}\right)
$$

On the other hand, we have

$$
\begin{aligned}
g\left(1-\epsilon_{0}\right) & <\mathcal{g}\left(F_{y_{m_{i}}, y_{n_{i}}, a}\left(t_{0}\right)\right) \\
& \leq \mathcal{g}\left(F_{y_{m_{i}}, y_{n_{i}}, y_{n_{i}+1}}\left(t_{0}\right)\right)+\mathcal{g}\left(F_{y_{m_{i}}, y_{n_{i}+1}, a}\left(t_{0}\right)\right)+\mathcal{g}\left(F_{y_{n_{i}+1}, y_{n_{i}}, a}\left(t_{0}\right)\right) .
\end{aligned}
$$

Now, consider $g\left(F_{y_{m_{i}}, y_{n_{i}+1}, a}\left(t_{0}\right)\right)$ in (1.28), assume that both $n_{i}$ and $m_{i}$ are even. Then 
by (iv), we have

$$
\begin{aligned}
& g\left(F_{y_{m_{i}}, y_{n_{i}+1}, a}\left(t_{0}\right)\right)=g\left(F_{A x_{m_{i}}, B x_{n_{i}+1}, a}\left(t_{0}\right)\right) \\
& \leq \phi\left(\operatorname { m a x } \left\{g\left(F_{S x_{m_{i}}, T x_{n_{i}+1}, a}\left(t_{0}\right)\right),\right.\right. \\
& \mathfrak{g}\left(F_{S x_{m_{i}}, A x_{m_{i}}, a}\left(t_{0}\right)\right), \mathfrak{g}\left(F_{T x_{n_{i}+1}, B x_{n_{i}+1}, a}\left(t_{0}\right)\right), \\
& \left.\left.\frac{1}{2}\left(g\left(F_{S x_{m_{i}}, B x_{n_{i}+1}, a}\left(t_{0}\right)\right)+g\left(F_{T x_{n_{i}+1}, B x_{n_{i}+1}, a}\left(t_{0}\right)\right)\right)\right\}\right) \\
& =\phi\left(\operatorname { m a x } \left\{g\left(F_{y_{m_{i}-1}, y_{n_{i}}, a}\left(t_{0}\right)\right)\right.\right. \text {, } \\
& \boldsymbol{g}\left(F_{y_{m_{i}-1}, y_{m_{i}}, a}\left(t_{0}\right)\right), \mathcal{g}\left(F_{y_{n_{i}}, y_{n_{i}+1}, a}\left(t_{0}\right)\right), \\
& \left.\left.\frac{1}{2}\left(g\left(F_{y_{m_{i}-1}, y_{n_{i}+1}, a}\left(t_{0}\right)\right)+g\left(F_{y_{n_{i}}, y_{m_{i}}, a}\left(t_{0}\right)\right)\right)\right\}\right) .
\end{aligned}
$$

By (1.27), (1.28), and (1.29), letting $i \rightarrow \infty$ in (1.29), we have

$$
g\left(1-\epsilon_{0}\right) \leq \phi\left(\max \left\{g\left(1-\epsilon_{0}\right), 0,0, g\left(1-\epsilon_{0}\right)\right\}\right)=\phi\left(g\left(1-\epsilon_{0}\right)\right)<g\left(1-\epsilon_{0}\right),
$$

which is a contradiction. Therefore, $\left\{y_{n}\right\}$ is a Cauchy sequence in $X$.

Now, we prove our main theorem.

If we prove $\lim _{n \rightarrow \infty} g\left(F_{y_{n}, y_{n+1}, a}(t)\right)=0$ for all $t>0$, then by Lemma 1.19, the sequence $\left\{y_{n}\right\}$ defined by (1.24) is a Cauchy sequence in $X$.

First we prove $\lim _{n \rightarrow \infty} g\left(F_{y_{n}, y_{n+1}, a}(t)\right)=0$ for all $t>0$. In fact, by Theorem 1.18(iv) and (1.24), we have

$$
\begin{aligned}
& g\left(F_{y_{2 n}, y_{2 n+1}, a}(t)\right)=g\left(F_{A x_{2 n}, B x_{2 n+1}, a}(t)\right) \\
& \leq \phi\left(\operatorname { m a x } \left\{g\left(F_{S x_{2 n}, T x_{2 n+1}, a}(t)\right),\right.\right. \\
& g\left(F_{S x_{2 n}, A x_{2 n}, a}(t)\right), g\left(F_{T x_{2 n}, B x_{2 n+1}, a}(t)\right), \\
& \left.\left.\frac{1}{2}\left(g\left(F_{S x_{2 n}, B x_{2 n+1}, a}(t)\right)+g\left(F_{T x_{2 n+1}, A x_{2 n}, a}(t)\right)\right)\right\}\right) \\
& =\phi\left(\operatorname { m a x } \left\{g\left(F_{y_{2 n-1}, y_{2 n}, a}(t)\right)\right.\right. \text {, } \\
& g\left(F_{y_{2 n-1}, y_{2 n}, a}(t)\right), g\left(F_{y_{2 n}, y_{2 n+1}, a}(t)\right), \\
& \left.\left.\frac{1}{2}\left(g\left(F_{y_{2 n-1}, y_{2 n+1}, a}(t)\right)+g(1)\right)\right\}\right) \\
& \leq \phi\left(\operatorname { m a x } \left\{g\left(F_{y_{2 n-1}, y_{2 n}, a}(t)\right), g\left(F_{y_{2 n}, y_{2 n+1}, a}(t)\right)\right.\right. \text {, } \\
& \left.\left.g\left(F_{y_{2 n-1}, y_{2 n}, a}(t)\right)+\mathcal{g}\left(F_{y_{2 n}, y_{2 n+1}, a}(t)\right)\right\}\right)
\end{aligned}
$$

if $g\left(F_{y_{2 n-1}, y_{2 n}, a}(t)\right) \leq g\left(F_{y_{2 n}, y_{2 n+1}, a}(t)\right)$ for all $t>0$, then by Theorem 1.18(iv), $g\left(F_{y_{2 n}, y_{2 n+1}, a}(t)\right) \leq \phi\left(g\left(F_{y_{2 n}, y_{2 n+1}, a}(t)\right)\right)$ and thus, by Lemma 1.8, $g\left(F_{y_{2 n}, y_{2 n+1}, a}(t)\right)=0$ for all $t>0$. Similarly, we have $g\left(F_{y_{2 n+1}, y_{2 n+2}, a}(t)\right)=0$, thus we have $\lim _{n \rightarrow \infty} g\left(F_{y_{n}, y_{n+1}, a}(t)\right)$ $=0$ for all $t>0$. On the other hand, if $g\left(F_{y_{2 n-1}, y_{2 n}, a}(t)\right) \geq g\left(F_{y_{2 n}, y_{2 n+1}, a}(t)\right)$, then by Theorem 1.18(iv), we have

$$
g\left(F_{y_{2 n}, y_{2 n+1}, a}(t)\right) \leq \phi\left(g\left(F_{y_{2 n-1}, y_{2 n}, a}(t)\right)\right) \quad \forall t>0 .
$$


Similarly,

$$
g\left(F_{y_{2 n+1}, y_{2 n+2}, a}(t)\right) \leq \phi\left(g\left(F_{y_{2 n}, y_{2 n+1}, a}(t)\right)\right) \quad \forall t>0
$$

hence

$$
g\left(F_{y_{n}, y_{n+1}, a}(t)\right) \leq \phi\left(g\left(F_{y_{n-1}, y_{n}, a}(t)\right)\right) \quad \forall t>0, n=1,2,3, \ldots,
$$

therefore by Lemma 1.8 ,

$$
\lim _{n \rightarrow \infty} g\left(F_{y_{n}, y_{n+1}, a}(t)\right)=0 \quad \forall t>0,
$$

which implies that $\left\{y_{n}\right\}$ is a Cauchy sequence in $X$ by Lemma 1.19. Since $(X, F, \Delta)$ is complete, the sequence $\left\{y_{n}\right\}$ converges to a point $z \in X$ and so the subsequences $\left\{A x_{2 n}\right\},\left\{B x_{2 n+1}\right\},\left\{S x_{2 n}\right\}$, and $\left\{T x_{2 n+1}\right\}$ of $\left\{y_{n}\right\}$ also converge to the limit $z$.

Now, suppose that $T$ is continuous. Since $B$ and $T$ are weak compatible of type $(A)$, by Proposition 1.17, BT $x_{2 n+1}, T T x_{2 n+1}$ tend to $T z$ as $n$ tends to $\infty$. Putting $x=x_{2 n}$ and $y=T x_{2 n+1}$ in Theorem 1.18(iv), we have

$$
\begin{aligned}
g\left(F_{A x_{2 n}, B T x_{2 n+1}, a}(t)\right) & \\
\leq \phi(\max & \left\{g\left(F_{S x_{2 n}, T T x_{2 n+1}, a}(t)\right), g\left(F_{S x_{2 n}, A x_{2 n}, a}(t)\right), g\left(F_{T T x_{2 n+1}, B T x_{2 n+1}, a}(t)\right),\right. \\
& \left.\left.\frac{1}{2}\left(g\left(F_{S x_{2 n}, B T x_{2 n+1}, a}(t)\right)+g\left(F_{T T x_{2 n+1}, A x_{2 n}, a}(T)\right)\right)\right\}\right) \quad \forall t>0 .
\end{aligned}
$$

Letting $n \rightarrow \infty$ in (1.36), we get

$$
\begin{aligned}
g\left(F_{z, T z, a}(t)\right) \leq & \phi\left(\operatorname { m a x } \left\{g\left(F_{z, T z, a}(t)\right), g\left(F_{z, z, a}(t)\right), g\left(F_{T z, T z, a}(t)\right),\right.\right. \\
& \left.\left.\frac{1}{2}\left(g\left(F_{z, T z, a}(t)\right)+g\left(F_{T z, z, a}(t)\right)\right)\right\}\right) \\
= & \phi\left(g\left(F_{z, T z, a}(t)\right)\right) \quad \forall t>0,
\end{aligned}
$$

which means that $g\left(F_{z, T z, a}(t)\right)=0$ for all $t>0$ by Lemma 1.8 and so we have $T z=z$. Again replacing $x$ by $x_{2 n}$ and $y$ by $z$ in Theorem 1.18(iv), we have

$$
\begin{array}{r}
g\left(F_{A x_{2 n}, B z, a}(t)\right) \leq \phi\left(\operatorname { m a x } \left\{g\left(F_{S x_{2 n}, T z, a}(t)\right), \mathcal{g}\left(F_{S x_{2 n}, A x_{2 n}, a}(t)\right), g\left(F_{T z, B z, a}(t)\right),\right.\right. \\
\left.\left.\frac{1}{2}\left(g\left(F_{S x_{2 n}, B z, a}(t)\right)+g\left(F_{T z, A x_{2 n}, a}(t)\right)\right)\right\}\right) \quad \forall t>0 .
\end{array}
$$

Letting $n \rightarrow \infty$ in (1.38), we get

$$
\begin{aligned}
g\left(F_{z, B z, a}(t)\right) \leq \phi(\max \{ & g\left(F_{z, z, a}(t)\right), \mathcal{g}\left(F_{z, B z, a}(t)\right), \mathcal{g}\left(F_{z, B z, a}(t)\right), \\
& \left.\left.\frac{1}{2}\left(g\left(F_{z, B z, a}(t)\right)+g\left(F_{z, z, a}(t)\right)\right)\right\}\right) \quad \forall t>0,
\end{aligned}
$$

which implies that $g\left(F_{z, B z, a}(t)\right) \leq \phi\left(g\left(F_{z, B z, a}(t)\right)\right)$ for all $t>0$ and so we have $B z=$ $z$. Since $B(x) \subset S(X)$, there exists a point $w \in X$ such that $B z=S w=z$. By using condition Theorem 1.18(iv) again, we have

$$
\begin{aligned}
g\left(F_{A w, z, a}(t)\right) & =g\left(F_{A w, B z, a}(t)\right) \\
\leq & \phi\left(\operatorname { m a x } \left\{\left(F_{S w, T z, a}(t)\right), g\left(F_{S w, A w, a}(t)\right), g\left(F_{T z, B z, a}(t)\right),\right.\right. \\
& \left.\left.\quad \frac{1}{2}\left(g\left(F_{S w, B z, a}(t)\right)+g\left(F_{T z, A w, a}(t)\right)\right)\right\}\right) \\
& \leq \phi\left(g\left(F_{A w, z, a}(t)\right)\right) \quad \forall t>0,
\end{aligned}
$$


which means that $A w=z$. Since $A$ and $S$ are weak compatible mappings of type (A) and $A w=S w=z$, by Proposition 1.16, $A z=A S w=S S w=S z$. Again by using Theorem 1.18(iv), we have $A z=z$.

Therefore, $A z=B z=S z=T z=z$, that is, $z$ is a common fixed point of the given mappings $A, B, S, T$. The uniqueness of the common fixed point $z$ follows easily from Theorem 1.18(iv).

REMARK 1.20. In Theorem 1.18, if $S$ and $T$ are continuous, then by Proposition 1.15 , the theorem is true even though the pairs $A, S$ and $B, T$ are compatible of type (A) instead of the condition (ii).

\section{Application}

THEOREM 1.21. Let $(X, F, t)$ be a complete $2 N A$ Menger PM-space and $A, B, S$, and $T$ be the mappings from the product $X \times X$ to $X$ such that

$$
\begin{gathered}
A(X \times\{y\}) \subseteq T(X \times\{y\}), \quad B(X \times\{y\}) \subseteq S(X \times\{y\}), \\
g\left(F_{A(T(x, y), y), T(A(x, y), y), a}(t)\right) \leq g\left(F_{A(x, y), T(x, y), a}(t)\right), \\
g\left(F_{B(S(x, y), y), S(B(x, y), y), a}(t)\right) \leq g\left(F_{B(x, y), S(x, y), a}(t)\right),
\end{gathered}
$$

for all $t>0$. If $S$ and $T$ are continuous with respect to their direct argument and

$$
\begin{aligned}
& g\left(F_{A(x, y), B\left(x^{\prime}, y^{\prime}\right), a}(t)\right) \\
& \leq \phi(\max \{ g\left(F_{S(x, y), T\left(x^{\prime}, y^{\prime}\right), a}(t)\right), \\
& g\left(F_{S(x, y), A(x, y), a}(t)\right), g\left(F_{T\left(x^{\prime}, y^{\prime}\right), B\left(x^{\prime}, y^{\prime}\right), a}(t)\right), \\
&\left.\left.\frac{1}{2}\left(g\left(F_{S(x, y), B\left(x^{\prime}, y^{\prime}\right), a}(t)\right)+g\left(F_{T\left(x^{\prime}, y^{\prime}\right), A(x, y), a}(t)\right)\right)\right\}\right)
\end{aligned}
$$

for all $t>0$ and $x, y, x^{\prime}, y^{\prime}$ in $X$, then there exists only one point $b$ in $X$ such that

$$
A(b, y)=S(b, y)=B(b, y)=T(b, y) \quad \forall y \text { in } X
$$

Proof. By (1.42),

$$
\begin{aligned}
& g\left(F_{A(x, y), B\left(x^{\prime}, y^{\prime}\right), a}(t)\right) \\
& \leq \phi(\max \{ g\left(F_{S(x, y), T\left(x^{\prime}, y^{\prime}\right), a}(t)\right), \\
& g\left(F_{S(x, y), A(x, y), a}(t)\right), g\left(F_{T\left(x^{\prime}, y^{\prime}\right), B\left(x^{\prime}, y^{\prime}\right), a}(t)\right), \\
&\left.\left.\frac{1}{2}\left(g\left(F_{S(x, y), B\left(x^{\prime}, y^{\prime}\right), a}(t)\right)+g\left(F_{T\left(x^{\prime}, y^{\prime}\right), A(x, y), a}(t)\right)\right)\right\}\right)
\end{aligned}
$$

for all $t>0$, therefore by Theorem 1.18 , for each $y$ in $X$, there exists only one $x(y)$ in $X$ such that

$$
A(x(y), y)=S(x(y), y)=B(x(y), y)=T(x(y), y)=x(y)
$$


for every $y, y^{\prime}$ in $X$

$$
\begin{aligned}
g\left(F_{x(y), x\left(y^{\prime}\right), a}(t)\right)= & g\left(F_{A(x(y), y), A\left(x\left(y^{\prime}\right), y^{\prime}\right), a}(t)\right) \\
\leq & \phi\left(\operatorname { m a x } \left\{g\left(F_{A(x, y), A\left(x, y^{\prime}\right), a}(t)\right),\right.\right. \\
& \quad g\left(F_{A(x, y), A(x, y), a}(t)\right), g\left(F_{T\left(x^{\prime}, y^{\prime}\right), A\left(x^{\prime}, y^{\prime}\right), a}(t)\right), \\
& \left.\left.\frac{1}{2}\left(g\left(F_{A(x, y), A\left(x^{\prime}, y^{\prime}\right), a}(t)\right)+g\left(F_{A\left(x^{\prime}, y^{\prime}\right), A(x, y), a}(t)\right)\right)\right\}\right) \\
= & g\left(F_{x(y), x\left(y^{\prime}\right), a}(t)\right) .
\end{aligned}
$$

This implies that $x(y)=x\left(y^{\prime}\right)$ and hence $x(\cdot)$ is some constant $b \in X$ so that

$$
A(b, y)=b=T(b, y)=S(b, y)=B(b, y) \quad \forall y \text { in } X
$$

\section{REFERENCES}

[1] Y. J. Cho, K. S. Ha, and S.-S. Chang, Common fixed point theorems for compatible mappings of type (A) in non-Archimedean Menger PM-spaces, Math. Japon. 46 (1997), no. 1, 169-179. CMP 1466 131. Zbl 888.47038.

Renu Chugh: Department of Mathematics, Maharshi Dayanand University, ROHTAK-124001, INDIA

E-mail address: anujtele@ndb.vsn 1. net. in

Sumitra: Department of MATHEMATICS, MAHARShi DAYANAND UNIVERSity, ROHTAK124001, INDIA

E-mail address: mastak@de12.vsn1.net.in 


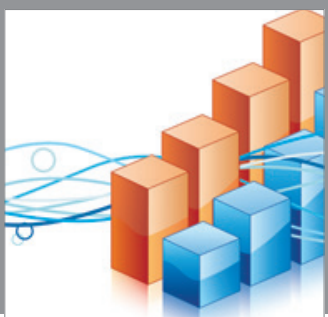

Advances in

Operations Research

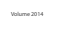

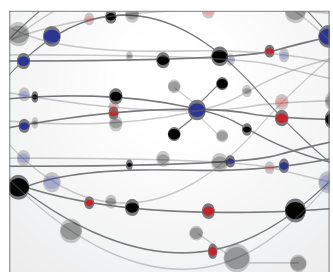

\section{The Scientific} World Journal
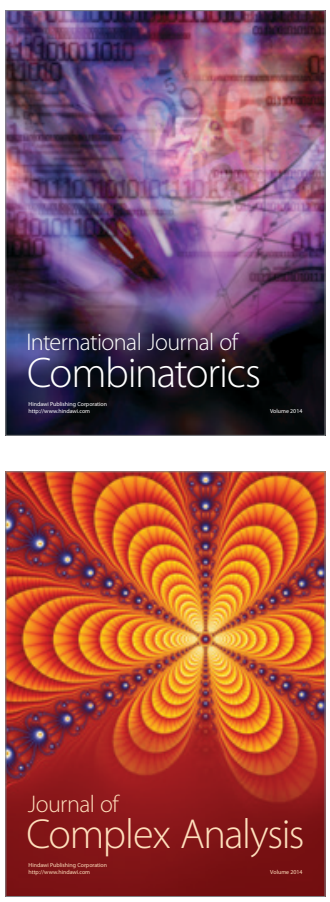

International Journal of

Mathematics and

Mathematical

Sciences
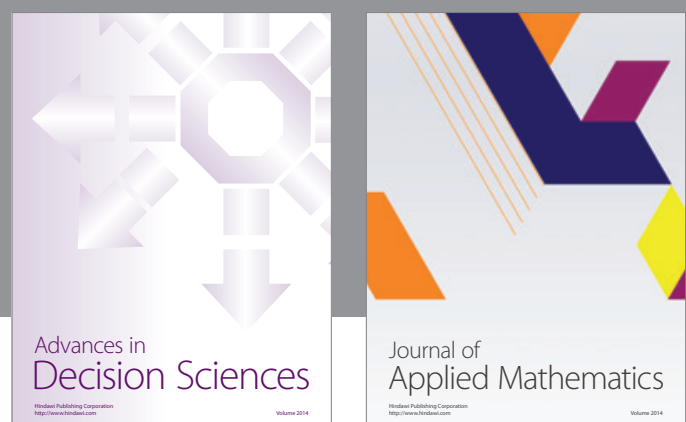

Journal of

Applied Mathematics
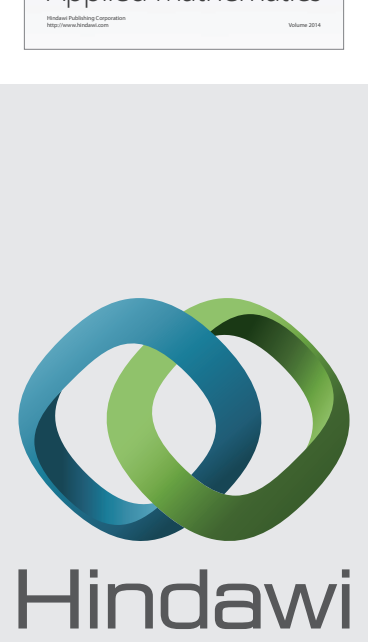

Submit your manuscripts at http://www.hindawi.com
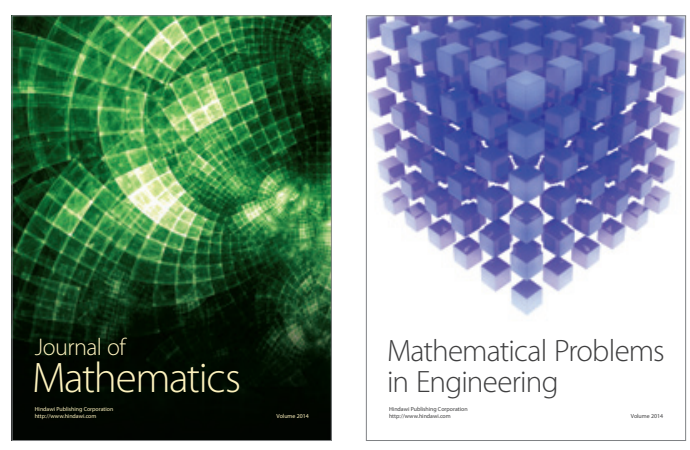

Mathematical Problems in Engineering
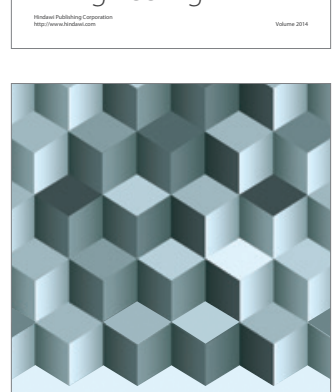

Journal of

Function Spaces
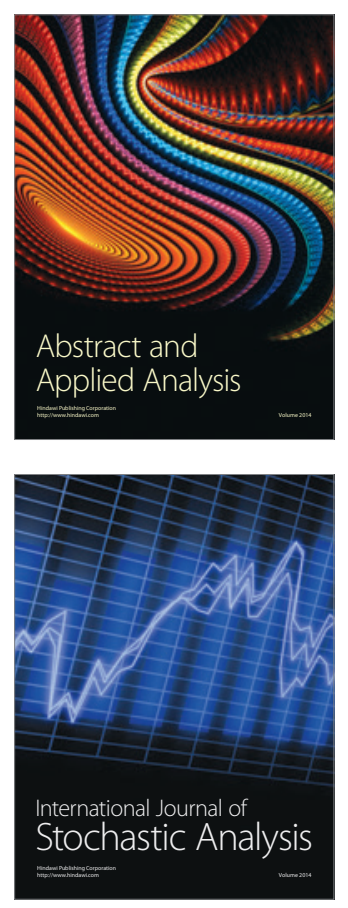

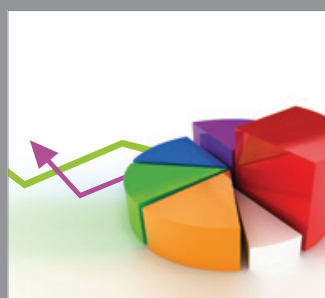

ournal of

Probability and Statistics

Promensencen
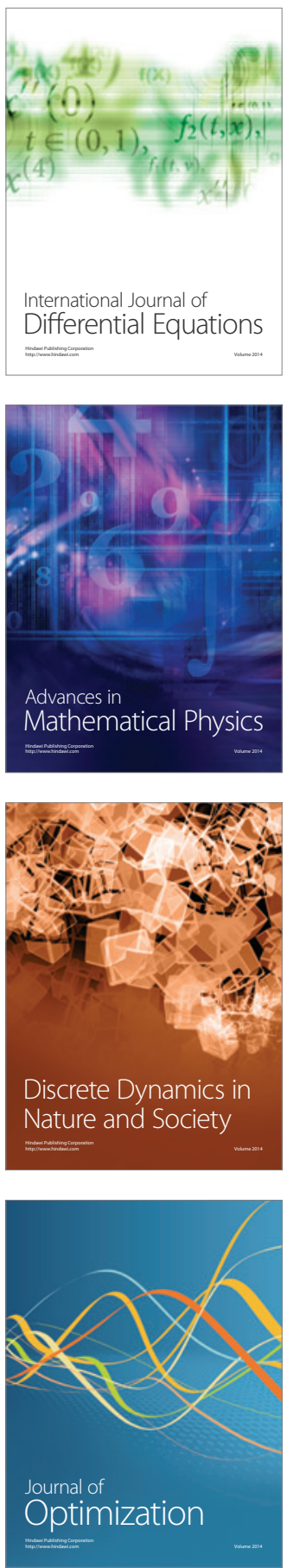